\title{
Comparison of the clinicopathological behavior of the follicular variant of papillary thyroid carcinoma and classical papillary thyroid carcinoma: A systematic review and meta-analysis
}

\author{
JING YANG ${ }^{1}$, YANPING GONG ${ }^{1}$, SHUPING YAN $^{1}$, QINGQUAN SHI $^{2}$, JINGQIANG ZHU $^{1}$, \\ ZHIHUI LI ${ }^{1}$, QIANG CHEN ${ }^{1}$ and RIXIANG GONG ${ }^{1}$ \\ ${ }^{1}$ Department of Thyroid and Breast Surgery, West China Hospital; ${ }^{2}$ Department of Obstetrics and Gynecology, \\ West China Second University Hospital, Sichuan University, Chengdu, Sichuan 610041, P.R. China
}

Received October 16, 2014; Accepted February 25, 2015

DOI: $10.3892 / \mathrm{mco} .2015 .540$

\begin{abstract}
The follicular variant of papillary thyroid carcinoma (FV-PTC) is the second most common type of papillary thyroid carcinoma (PTC), and it has been increasingly diagnosed in recent years. However, whether FV-PTC behaves differently from classical PTC (C-PTC) remains controversial. To address this controversy, a meta-analysis was performed to determine the potential differences between FV-PTC and C-PTC in their clinicopathological behavior. The relevant published studies between January 1, 2003 and August 31, 2014 were reviewed according to the defined selection criteria using the PubMed database. Review Manager was used to calculate the pooled odds ratio (OR) or the mean difference (MD) with a 95\% confidence interval (CI), using a random- or fixed-effect model for all analyses. In total, 112 studies were identified and examined; finally, only 36 studies met the inclusion criteria. In the 36 studies, compared to the clinicopathological behavior of patients with C-PTC, patients with FV-PTC had the following parameters: Similar mean age and similar prevalence of gender, tumor size $\geq 10 \mathrm{~mm}$, multifocality, capsular invasion, vascular invasion, lymphocytic and/or Hashimoto's thyroiditis, and clinical stage; a larger mean tumor size and higher prevalence of age $\geq 45$ years; and lower prevalence of extrathyroidal extensions, lymph node metastases, $B R A F$ mutation and recurrence. The meta-analysis suggested that patients with FV-PTC have a more favorable clinicopathological behavior and improved prognosis compared to patients with C-PTC. Thus, patients with FV-PTC and C-PTC may be managed differently, and
\end{abstract}

Correspondence to: Professor Rixiang Gong, Department of Thyroid and Breast Surgery, West China Hospital, Sichuan University, 37 Guo Xue Xiang, Chengdu, Sichuan 610041, P.R. China

E-mail: gongrixiang@scu.edu.cn

Key words: thyroid carcinoma, papillary, follicular, classical, meta-analysis the two types of PTC should be clearly distinguished in future retrospective or prospective studies.

\section{Introduction}

Well-differentiated thyroid cancer is the most common endocrine tumor, and its prevalence is increasing worldwide (1). The majority of thyroid cancer cases $(\sim 85 \%)$ are papillary thyroid carcinoma (PTC) (2). A number of PTC variants have been described, including classical, follicular, oncocytic, solid, tall cell, columnar cell, diffuse sclerosing and cribriform $(3,4)$. Among these variants, the conventional or classical type is the most common and accounts for $\leq 54.2 \%$ of all PTC cases in certain reported series (5-8). The follicular variant is the second most common subtype and constitutes 4.9-41.2\% PTC cases in different series (7-12). Since the follicular variant of PTC (FV-PTC) was first described by Crile and Hazard in 1953 (13), FV-PTC has been increasingly diagnosed and accounts for $41-53 \%$ of PTC cases $(7,14)$.

As FV-PTC exhibits a mixed histopathological picture of PTC and follicular thyroid carcinoma (FTC), certain investigators have hypothesized that FV-PTC has specific characteristics from the two types. Numerous studies have since investigated the clinical behavior of FV-PTC and the classical type of PTC (C-PTC) and compared the two; however, certain results were contradictory. In different studies, the incidence of aggressive clinical features was identified as higher, similar or lower in FV-PTC compared to C-PTC $(5,7,8,10,12,15-20)$.

Meta-analysis is a powerful tool for summarizing the results of different studies by producing a single estimate of the major effect with enhanced precision. A major advantage of a meta-analysis is the increase in sample size, which may reduce the probability that a random error will produce false-positive or false-negative associations.

A meta-analysis was performed to quantify and compare the clinical parameters of C-PTC and FV-PTC and provide some guidance on the management and prognosis of FV-PTC. The following clinicopathological parameters were evaluated: Age, gender, tumor size, multifocality, capsular invasion, vascular invasion, extrathyroidal extension, lymph 
node metastasis, lymphocytic and/or Hashimoto's thyroiditis, clinical stage, $B R A F$ mutation and recurrence.

\section{Materials and methods}

Selection criteria. Studies that examined the associations of FV-PTC and C-PTC with clinicopathological parameters were searched for. The following criteria were considered when selecting the studies: i) Studies published in English between January 1, 2003 and August 31, 2014. ii) The criteria of C-PTC include classical, conventional and pure PTC. iii) Clinicopathological parameters with detailed data on C-PTC and FV-PTC tissue were included from the same studies that assessed different types of carcinoma, such as primary, follicular, anaplastic and medullary carcinomas. iv) Only studies analyzing at least two of the above categories of clinicopathological data and containing $\geq 5$ cases reported FV-PTC and C-PTC. v) When multiple studies were published by the same investigators or groups, the newest or most informative single study was selected. The following studies were excluded: i) Review studies without original data; ii) absent or inappropriately reported clinicopathological data; iii) single or pure case reports; iv) animal research; and v) familial research studies.

Collection of published studies. A literature search was carried out using the PubMed database (http://www.ncbi.nlm. nih.gov/pubmed). The search term combination was 'follicular variant of papillary thyroid carcinoma' OR 'FV-PTC' OR 'FVPTC' OR 'FPTC' OR 'F-PTC.' Relevant studies were selected on the basis of the summary analysis. Any duplication of data was carefully avoided by examining the names of all the authors and different medical centers involved in each publication. Overlapping studies or data and studies that were unrelated to the meta-analysis were excluded. Two investigators (J. Yang and Y.P. Gong) used the EndNote reference tool to independently screen and select studies. All the procedures conformed to the guidelines for the meta-analysis of observational studies in epidemiology (21).

Data analyses and statistical methods. Review Manager (version 5.1; http://tech.cochrane.org/revman) was used to perform all the statistical analyses, including the calculation of the summary odds ratio (OR) or the mean difference (MD) with a 95\% confidence interval (CI), using a random- or fixed-effect model for all the analyses. The choice of each individual statistical method depended on whether the measured event was dichotomous or continuous, whereas the choice of a randomor fixed-effect model depended on the tests for heterogeneity. The heterogeneity of the studies was assessed using the $\chi^{2}$ test of heterogeneity and the $\mathrm{I}^{2}$ measure of inconsistency. When the heterogeneity in the $\chi^{2}$ test showed a P-value of $<0.10$ or when the $\mathrm{I}^{2}$ measure was $>50 \%$, the random-effect model was chosen, otherwise the fixed-effect model was used. The 95\% CI was constructed around the effect size to establish its significance.

For the OR of dichotomous events, if the $95 \%$ CI of an OR included 1, the two groups were not considered statistically different, otherwise they were considered statistically different. For the MD of continuous events, if the 95\% CI crossed the

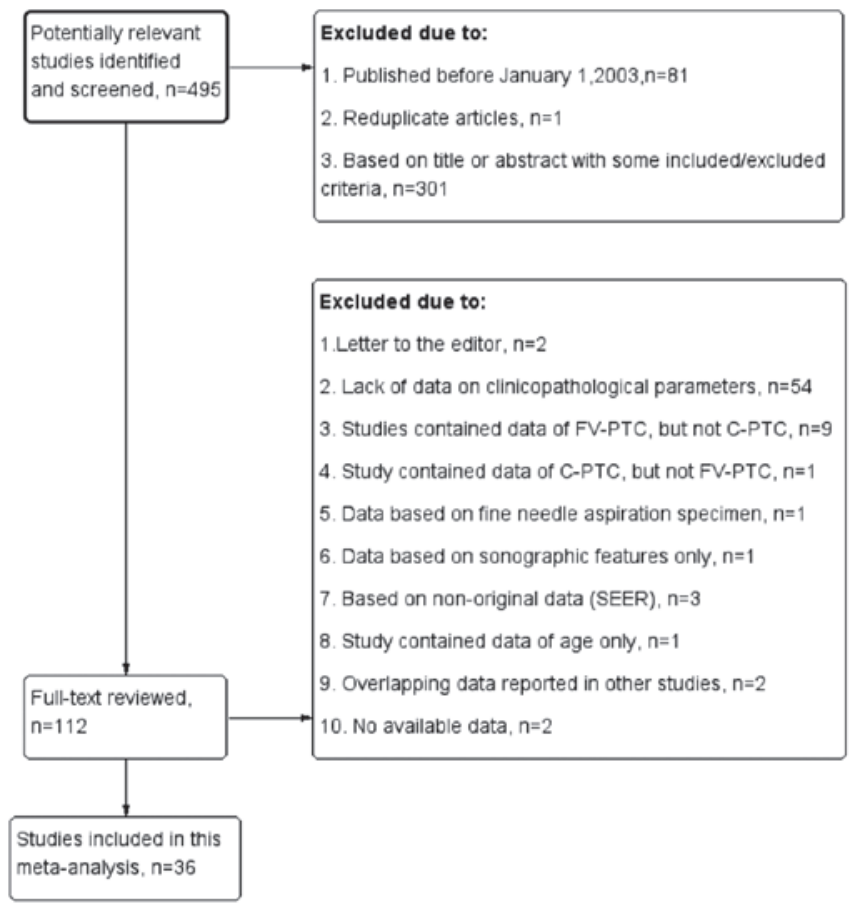

Figure 1. Study selection process. FV-PTC, follicular variant of papillary thyroid carcinoma. C-PTC, classical, conventional or pure papillary variant of papillary thyroid carcinoma; SEER, the Surveillance, Epidemiology and End Results database.

null point (zero), then the possibility that the difference should be attributed to chance could not be ruled out. When the null point fell outside the $95 \% \mathrm{CI}$ of an MD, the observed difference was considered statistically significant. The potential publication bias was assessed using Begg's funnel plot and Egger's test by Stata 12.0 software (Stata Corporation, College Station, TX, USA). $\mathrm{P}<0.05$ was considered to indicate a statistically significant difference.

\section{Results}

Clinicopathological parameters. Fig. 1 summarizes the study selection process. A total of 495 abstracts and titles were obtained using a PubMed search, of which 81 were published before January 1,2003 and 1 duplicate study was excluded. Of the remaining 413, 112 full-text studies were deemed relevant and were examined in detail. Eventually, following the application of all the inclusion and exclusion criteria, 36 studies $(7,8,10,12,14,16,17,19,20,22-48)$ fulfilled the eligibility criteria. The main features of the eligible studies are summarized in Table I. In each category of clinicopathological parameters, some heterogeneity was present. In terms of tumor size, multifocality, capsular invasion, vascular invasion, extrathyroidal extension and lymph node metastasis, their heterogeneity was assessed using $\chi^{2}$ tests and the $\mathrm{P}$-value was $<0.10$ (or $\mathrm{I}^{2}$ measures were $>50 \%$ ). The random-effect models were selected, but for the remaining parameters, fixed-effect models were used. The combined results of the meta-analysis and the heterogeneity test are shown in Table II.

Gender. In total, 33 studies were comparable in terms of gender. The prevalence of females among patients with FV-PTC 
Table I. Characteristics of individual studies included in the meta-analysis.

\begin{tabular}{|c|c|c|c|c|c|c|c|c|}
\hline \multirow[b]{2}{*}{ First author, year } & \multirow[b]{2}{*}{ Country } & \multicolumn{2}{|c|}{ Patients, no. } & \multicolumn{2}{|c|}{$\begin{array}{l}\text { Mean age (years) } \pm \text { SD } \\
\text { and/or } \geq(>) 45, \text { no. }(\%)\end{array}$} & \multicolumn{2}{|c|}{$\begin{array}{l}\text { Mean tumor size }(\mathrm{mm}) \pm \mathrm{SD} \\
\text { and/or } \geq(>) 10, \text { no. }(\%)\end{array}$} & \multirow[b]{2}{*}{ (Refs.) } \\
\hline & & FV-PTC & C-PTC & FV-PTC & C-PTC & FV-PTC & C-PTC & \\
\hline Abrosimov, 2007 & Japan & 34 & 148 & NA & NA & $>10 \pm 11(32.4)$ & $>10 \pm 71(47.3)$ & (22) \\
\hline Brzezianska, 2007 & Poland & 8 & 14 & $\begin{aligned} & 49(20.4) \\
\geq & 45 \pm 5(62.5)\end{aligned}$ & $\begin{aligned} & 47.57(17) \\
\geq & 45 \pm 7(50.0)\end{aligned}$ & NA & NA & $(23)$ \\
\hline Burningham, 2005 & USA & 46 & 114 & $46(17)^{\mathrm{b}}$ & $47(26)^{b}$ & $15(19)^{\mathrm{b}}$ & $10(16)^{b}$ & (19) \\
\hline Chang, 2006 & China & 85 & 170 & NA & NA & $27(18.4)$ & $23(13.0)$ & $(10)$ \\
\hline Costa, 2008 & Portugal & 17 & 16 & $37(10)$ & $45(25)$ & $34(25)$ & $35(15)$ & $(24)$ \\
\hline Daglar-Aday, 2013 & Turkey & 36 & 72 & $44.94(15.21)$ & $52.88(17.11)$ & NA & NA & $(25)$ \\
\hline Darr, 2011 & USA & 6 & 7 & $43(15)$ & $44(14)$ & $>20 \pm 5(16.7)$ & $>20 \pm 6(14.3)$ & $(26)$ \\
\hline Dettmer, 2013 & USA & 17 & 27 & $50.9(17.73)$ & $47.8(15.58)$ & NA & NA & $(27)$ \\
\hline Di Cristofaro, 2006 & France & 24 & 26 & $38.5(13)$ & $43.3(17.3)$ & $21.5(8.5)$ & $25.7(11.3)$ & $(28)$ \\
\hline Eloy, 2011 & Portugal & 31 & 42 & $\begin{array}{l}42.73(12.94) \\
\geq 45 \pm 15(48.4)\end{array}$ & $\begin{array}{c}39(17.75) \\
\geq 45 \pm 14(33.3)\end{array}$ & $\begin{array}{c}22.3(18) \\
>10 \pm 23(74.2)\end{array}$ & $\begin{array}{c}22.4(17.1) \\
>10 \pm 32(76.2)\end{array}$ & $(29)$ \\
\hline Ertek, 2012 & Turkey & 56 & 42 & 39.1 (10.6) & $46.7(12.9)$ & 34.7 (31.7) & $13.9(12.2)$ & $(14)$ \\
\hline Espadinha, 2009 & Portugal & 17 & 21 & $\begin{array}{l}35.41(25.37) \\
\geq 45 \pm 6(35.3)\end{array}$ & $\begin{array}{r}39.24(25.17) \\
\geq 45 \pm 14(33.3)\end{array}$ & NA & NA & $(30)$ \\
\hline Gao, 2012 & China & 25 & 84 & $\geq 45 \pm 13(52)$ & $\geq 45 \pm 54(64.3)$ & $>10 \pm 20(80)$ & $>10 \pm 61(72.6)$ & $(31)$ \\
\hline Hagag, 2006 & Israel & 92 & 99 & $\begin{array}{c}46(19.18) \\
\geq 45 \pm 52(56.5)\end{array}$ & $\begin{array}{c}44(9.95) \\
\geq 45 \pm 54(54.5)\end{array}$ & $22(19.2)$ & $20(9.9)$ & $(20)$ \\
\hline Hunt, 2004 & USA & 16 & 8 & $\begin{array}{l}50.38(11.63) \\
\geq 45 \pm 11(68.8)\end{array}$ & $\begin{array}{l}46.75(11.5) \\
\geq 45 \pm 5(62.5)\end{array}$ & $\begin{array}{c}30.1(23.7) \\
>10 \pm 11(68.8)\end{array}$ & $\begin{array}{l}15.6(13.7) \\
>10 \pm 4(50)\end{array}$ & $(32)$ \\
\hline Igci, 2013 & Turkey & 10 & 11 & $\begin{array}{l}38.5(12.75) \\
\geq 45 \pm 5(50)\end{array}$ & $\begin{array}{l}50.09(19.61) \\
\geq 45 \pm 4(36.4)\end{array}$ & $\begin{array}{l}30.6(16.9) \\
>10 \pm 9(90)\end{array}$ & $\begin{array}{c}30.6(16.6) \\
>10 \pm 11(100)\end{array}$ & (33) \\
\hline Igci, 2014 & Turkey & 25 & 15 & $\begin{array}{l}46.76(13.72) \\
\geq 45 \pm 15(60)\end{array}$ & $\begin{array}{l}48.73(15.66) \\
\geq 45 \pm 9(60)\end{array}$ & $\begin{aligned} & 14.4(9.7) \\
\geq & 10 \pm 16(64)\end{aligned}$ & $\begin{array}{c}16.4(10) \\
\geq 10 \pm 9(60)\end{array}$ & $(34)$ \\
\hline Ito, 2008 & Japan & 100 & 1,313 & $>55 \pm 41(41)$ & $>55 \pm 403(30.7)$ & $>40 \pm 6(6)$ & $>40 \pm 158(12)$ & $(35)$ \\
\hline Lang, 2006 & China & 67 & 308 & $38.5(14-83)^{\mathrm{c}}$ & $42.0(11-81)^{\mathrm{c}}$ & $25(10-85)^{\mathrm{c}}$ & $25(10-100)^{\mathrm{c}}$ & (8) \\
\hline Lassalle, 2011 & France & 5 & 11 & $39(8.06)$ & $44.45(18.05)$ & $22.4(8.4)$ & $17.7(7.8)$ & $(36)$ \\
\hline Lee, 2011 & Korea & 30 & 30 & $49.27(12.8)$ & $47.83(9.65)$ & $17.4(9.6)$ & $9.7(6.4)$ & $(37)$ \\
\hline Lim, 2013 & Korea & 85 & 2,947 & $45(13-84)^{c}$ & $47(24-74)^{c}$ & $8.2(0.5-125)^{\mathrm{c}}$ & $10(2-65)^{\mathrm{c}}$ & $(38)$ \\
\hline Liu, 2010 & USA & 73 & 114 & NA & NA & NA & NA & (39) \\
\hline Min, 2013 & Korea & 58 & 312 & $>45 \pm 44(75.9)$ & $>45 \pm 172(55.1)$ & $>10 \pm 15$ (25.9) & $>10 \pm 101(32.4)$ & $(40)$ \\
\hline Nechifor-Boila, 2013 & Romania & 90 & 98 & $27(6.1)$ & $26.3(5.5)$ & $27(16)$ & $21(11)$ & $(41)$ \\
\hline Oler, 2009 & Brazil & 47 & 73 & $\geq 45 \pm 23(53.5)$ & $\geq 45 \pm 31(43.7)$ & $\geq 10 \pm 35(74.5)$ & $\geq 10 \pm 50(70.4)$ & $(42)$ \\
\hline Ozdemir, 2011 & Turkey & 90 & 354 & $43.98(12.46)$ & $45.82(12.24)$ & $\begin{array}{c}16.9(13.9) \\
>10 \pm 55(61.1)\end{array}$ & $\begin{array}{c}10.6(9.7) \\
>10 \pm 127(35.9)\end{array}$ & $(17)$ \\
\hline Passler, 2003 & Austria & 37 & 117 & $46.4(10.9-74.8)^{\mathrm{c}}$ & $47.5(18.1-79.3)^{\mathrm{c}}$ & $17.9(17.6)$ & $24.2(21)$ & $(12)$ \\
\hline Rivera, 2009 & USA & 63 & 43 & $>45 \pm 30(47.6)$ & $>45 \pm 14(32.6)$ & $\geq 40 \pm 22(35.5)$ & $\geq 40 \pm 2(4.7)$ & $(43)$ \\
\hline Schulten, 2012 & $\begin{array}{l}\text { Saudi } \\
\text { Arabia }\end{array}$ & 42 & 115 & NA & NA & NA & NA & $(44)$ \\
\hline Sheu, 2010 & Germany & 30 & 10 & $46.4(15.4)$ & $48.1(14.0)$ & $28.5(18.1)$ & $26.2(18.8)$ & $(45)$ \\
\hline Slosar, 2009 & USA & 60 & 37 & NA & NA & $\geq 10 \pm 56(93.3)$ & $\geq 10 \pm 30(81.1)$ & $(46)$ \\
\hline Trovisco, 2005 & Portugal & 54 & 69 & $41.5(19.11)$ & $37.2(17.44)$ & $27(13.6)$ & $32(21)$ & $(47)$ \\
\hline Wreesmann, 2004 & USA & 17 & 25 & $40(25-75)^{\mathrm{c}}$ & $41(20-77)^{\mathrm{c}}$ & $25(8-65)^{\mathrm{c}}$ & $20(6-45)^{c}$ & $(48)$ \\
\hline Yuksel, 2008 & Turkey & 41 & 158 & $>40 \pm 31(75.6)$ & $>40 \pm 37(23.4)$ & $16.5(10.8)$ & $13.4(10)$ & $(16)$ \\
\hline Zidan, 2003 & Israel & 100 & 143 & $44(17-81)^{\mathrm{c}}$ & $43(11-78)^{\mathrm{c}}$ & $35(3-100)^{c}$ & $34(4-90)^{c}$ & (7) \\
\hline
\end{tabular}


Table II. Meta-analyses of the clinicopathological parameters between FV-PTC and C-PTC.

\begin{tabular}{|c|c|c|c|c|c|c|c|c|c|c|}
\hline \multirow{2}{*}{$\begin{array}{l}\text { Clinicopathological } \\
\text { characteristics }\end{array}$} & \multirow{2}{*}{$\begin{array}{l}\text { Included } \\
\text { study, n }\end{array}$} & \multicolumn{3}{|c|}{ Heterogeneity test } & \multirow{2}{*}{$\begin{array}{l}\text { Effects model } \\
\text { selection }\end{array}$} & \multirow{2}{*}{$\begin{array}{l}\text { OR/MD } \\
\text { (95\% confidence } \\
\text { interval) }\end{array}$} & \multicolumn{2}{|c|}{$\begin{array}{l}\text { Combined } \\
\text { effect test }\end{array}$} & \multirow{2}{*}{$\begin{array}{l}\text { Statistical } \\
\text { significance }\end{array}$} & \multirow{2}{*}{$\frac{\begin{array}{l}\text { Egger's } \\
\text { test }\end{array}}{\text { P-value }}$} \\
\hline & & $\chi^{2}$ & P-value & $\mathrm{I}^{2}, \%$ & & & $\mathrm{Z}$ & $\overline{P \text {-value }}$ & & \\
\hline Female & 33 & 29.61 & 0.59 & 0 & Fixed & $1.09(0.93-1.29)$ & 1.06 & 0.29 & No & 0.707 \\
\hline \multicolumn{11}{|l|}{ Age, years } \\
\hline Mean & 19 & 31.36 & 0.03 & 43 & Fixed & $-0.61(-1.75-0.53)^{\mathrm{a}}$ & 1.05 & 0.29 & No & 0.413 \\
\hline$\geq 45$ & 11 & 8.90 & 0.54 & 0 & Fixed & $1.45(1.11-1.90)$ & 2.69 & $<0.01$ & Yes & 0.754 \\
\hline \multicolumn{11}{|l|}{ Tumor size, mm } \\
\hline Mean & 17 & 50.72 & $<0.01$ & 68 & Random & $2.88(0.26-5.51)^{\mathrm{a}}$ & 2.15 & 0.03 & Yes & 0.485 \\
\hline$\geq 10$ & 10 & 21.73 & 0.01 & 59 & Random & $1.25(0.77-2.03)$ & 0.92 & 0.36 & No & 0.473 \\
\hline Multifocality & 17 & 40.85 & $<0.01$ & 61 & Random & $0.88(0.64-1.24)$ & 0.77 & 0.44 & No & 0.522 \\
\hline CI & 6 & 14.60 & 0.01 & 66 & Random & $0.82(0.48-1.39)$ & 0.74 & 0.46 & No & 0.577 \\
\hline VI & 9 & 31.84 & $<0.01$ & 75 & Random & $1.38(0.56-3.42)$ & 0.69 & 0.49 & No & 0.555 \\
\hline $\mathrm{EE}$ & 18 & 56.75 & $<0.01$ & 70 & Random & $0.40(0.25-0.64)$ & 3.86 & $<0.01$ & Yes & 0.609 \\
\hline LNM & 23 & 65.94 & $<0.01$ & 67 & Random & $0.35(0.25-0.49)$ & 6.12 & $<0.01$ & Yes & 0.450 \\
\hline LT or/and HT & 9 & 13.94 & 0.08 & 43 & Fixed & $0.79(0.61-1,02)$ & 1.82 & 0.07 & No & 0.419 \\
\hline CS (I+II) & 12 & 10.29 & 0.50 & 0 & Fixed & $1.17(0.90-1.52)$ & 1.16 & 0.25 & No & 0.605 \\
\hline$B R A F$ mutation & 13 & 8.15 & 0.77 & 0 & Fixed & $0.19(0.15-0.24)$ & 13.34 & $<0.01$ & Yes & 0.247 \\
\hline Recurrence & 8 & 5.46 & 0.60 & 0 & Fixed & $0.52(0.34-0.80)$ & 2.98 & $<0.01$ & Yes & 0.991 \\
\hline
\end{tabular}

${ }^{\mathrm{a}} \mathrm{MD}$ with a 95\% confidence interval. FV-PTC, follicular variant of papillary thyroid carcinoma; C-PTC, classical papillary thyroid carcinoma; OR, odds ratio; MD, standardized mean difference; CI, capsular invasion; VI, vascular invasion; EE, extrathyroidal extension; LNM, lymph node metastasis; LT, lymphocytic thyroiditis; HT, Hashimoto's thyroiditis; CS, clinical stage.

and C-PTC was $80.1(1,136 / 1,418)$ and $81.9 \%(5,627 / 6,872)$, respectively, and the difference was not statistically significant (OR, 1.09; 95\% CI, 0.93-1.29; P=0.29; Fig. 2A). No statistical heterogeneity was detected among the studies $\left(\chi^{2}=29.61\right.$, $\left.\mathrm{P}=0.59, \mathrm{I}^{2}=0 \%\right)$.

Age. Nineteen studies included mean age in the patient clinical data. The mean age of patients with FV-PTC ranged from 27 to 50.9 years, whereas those of the patients with C-PTC ranged from 26.3 to 52.88 years, and the difference was not significant (MD, $-0.61 ; 95 \%$ CI, $-1.75-0.53$; $\mathrm{P}=0.29$; Fig. 2B). No statistical heterogeneity was identified among the studies $\left(\chi^{2}=31.36, \mathrm{P}=0.03, \mathrm{I}^{2}=43 \%\right)$. In addition, 11 studies presented the prevalence of patients aged $\geq 45$ years, which overall was $56.4(219 / 388)$ and $51.5 \%$ (371/720) in patients with FV-PTC and C-PTC, respectively, and the difference was statistically significant (OR, 1.45; 95\% CI, 1.11-1.90; $\mathrm{P}=0.007$; Fig. 2C). There was no significant statistical heterogeneity among the studies $\left(\chi^{2}=8.90, \mathrm{P}=0.54, \mathrm{I}^{2}=0 \%\right)$.

Tumor size. Seventeen studies presented clinical data that included mean tumor size. The mean tumor size of patients with FV-PTC ranged from 14.4 to $34.7 \mathrm{~mm}$, whereas that of patients with C-PTC ranged from 9.7 to $35.0 \mathrm{~mm}$; this difference was statistically significant (MD, 2.88; 95\% CI, 0.26-5.51; $\mathrm{P}=0.03$; Fig. 2D). Significant statistical heterogeneity was present among the studies $\left(\chi^{2}=50.73, \mathrm{P}<0.0001, \mathrm{I}^{2}=68 \%\right)$. Ten studies presented the prevalence of the tumor size of patients with FV-PTC and C-PTC being $\geq 10 \mathrm{~mm}$, which overall was
$63.4(251 / 396)$ and $45.8 \%(496 / 1,084)$, respectively, and the difference was not significant (OR, 1.25; 95\% CI, 0.77-2.03; $\mathrm{P}=0.36$; Fig. 2E). There was significant statistical heterogeneity among the studies $\left(\chi^{2}=21.73, \mathrm{P}=0.010, \mathrm{I}^{2}=59 \%\right)$.

Multifocality. In 17 studies that analyzed multifocality, the overall percentage of patients with multifocality in FV-PTC and C-PTC was $31.6(257 / 813)$ and $30.7 \%(1,411 / 4,593)$, respectively, and the difference was not statistically significant (OR, 0.88; 95\% CI, 0.64-1.22; $\mathrm{P}=0.44 ;$ Fig. $2 \mathrm{~F})$. No statistical heterogeneity was detected among the studies $\left(\chi^{2}=40.85, \mathrm{P}=0.0006, \mathrm{I}^{2}=61 \%\right)$.

Capsular invasion. In 6 studies that assessed the capsular invasion of the tumor, the percentages of patients with capsular invasion in FV-PTC and C-PTC were 28.1 (115/409) and 30.2\% (281/932), respectively, and the difference was not statistically significant difference (OR, 0.82; 95\% CI, 0.48-1.39; $\mathrm{P}=0.46$; Fig. 2G). There was no statistical heterogeneity among the studies $\left(\chi^{2}=14.60, \mathrm{P}=0.01, \mathrm{I}^{2}=66 \%\right)$.

Vascular invasion. In 9 studies, the percentages of cases with vascular invasion in FV-PTC and C-PTC were reported; these were $19.8(85 / 430)$ and $12.2 \%$ (86/704), respectively, and the difference was not significant (OR, 1.38; 95\% CI, 0.56-3.42; $\mathrm{P}=0.49$; Fig. $2 \mathrm{H}$ ). Significant statistical heterogeneity was detected among the studies $\left(\chi^{2}=31.84, \mathrm{P}<0.0001, \mathrm{I}^{2}=75 \%\right)$.

Extrathyroidal extension. Eighteen studies presented the prevalence of cases with extrathyroidal extension in FV-PTC 
A

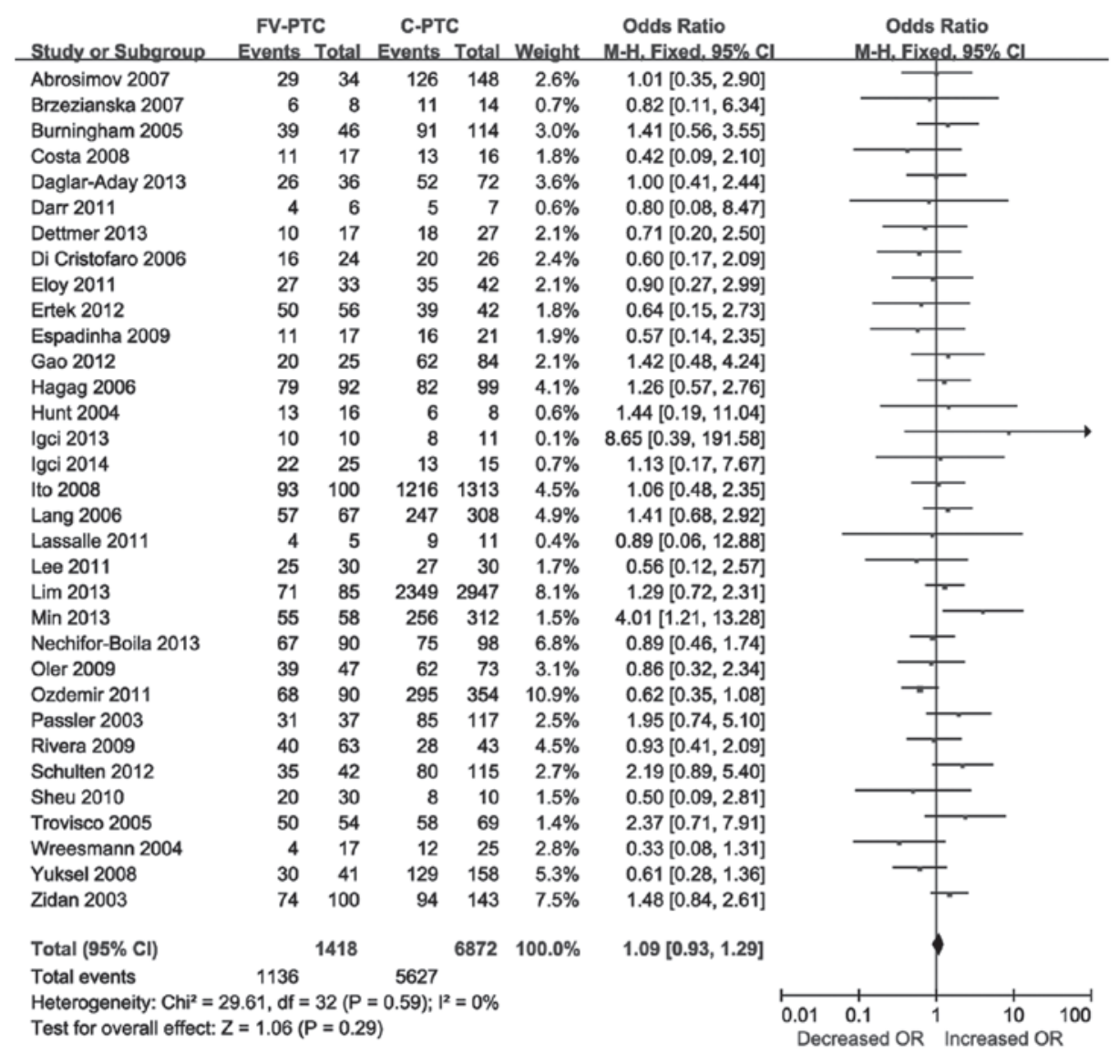

B

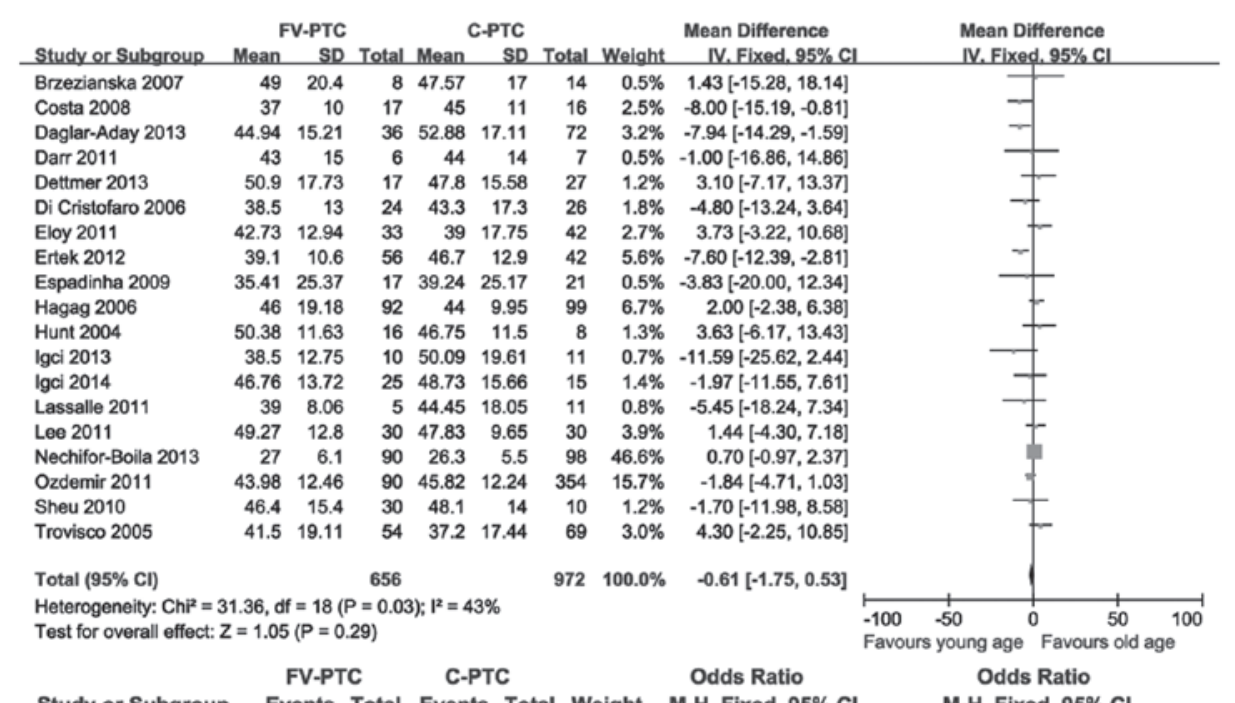

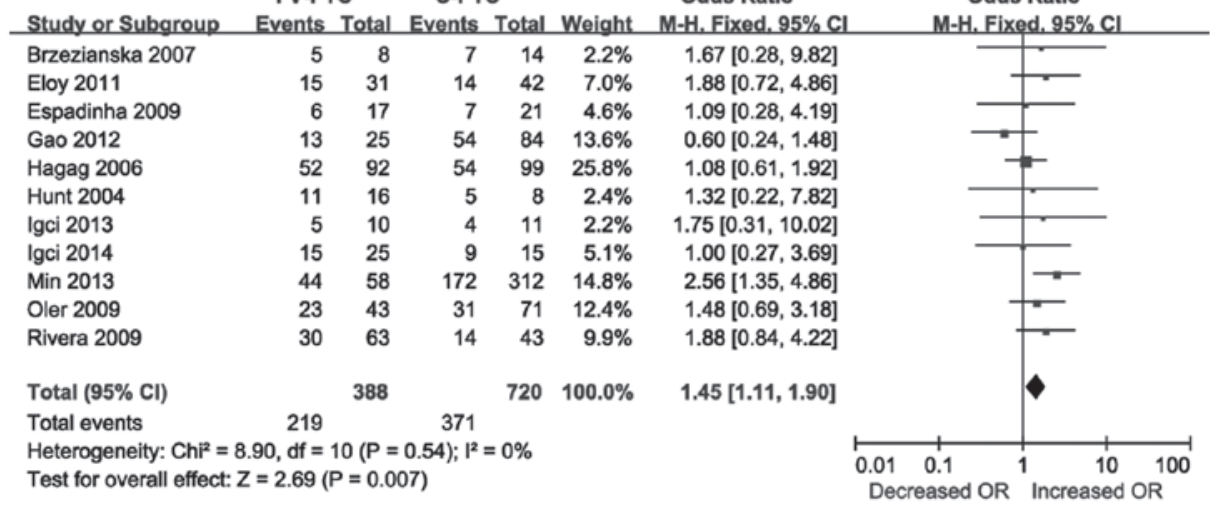

Figure 2. Forest plot for the comparison of (A) female, (B) mean age and (C) aged $\geq 45$ years between follicular variant of papillary thyroid carcinoma (FV-PTC) and classical papillary thyroid carcinoma (C-PTC). ORs or MDs with corresponding 95\% CIs of individual studies for comparison of clinicopathological characteristics are shown. The forest plot shows the effect size and 95\% CIs for each study and overall. OR, odds ratio; MD, mean difference; CI, confidence interval. 
D

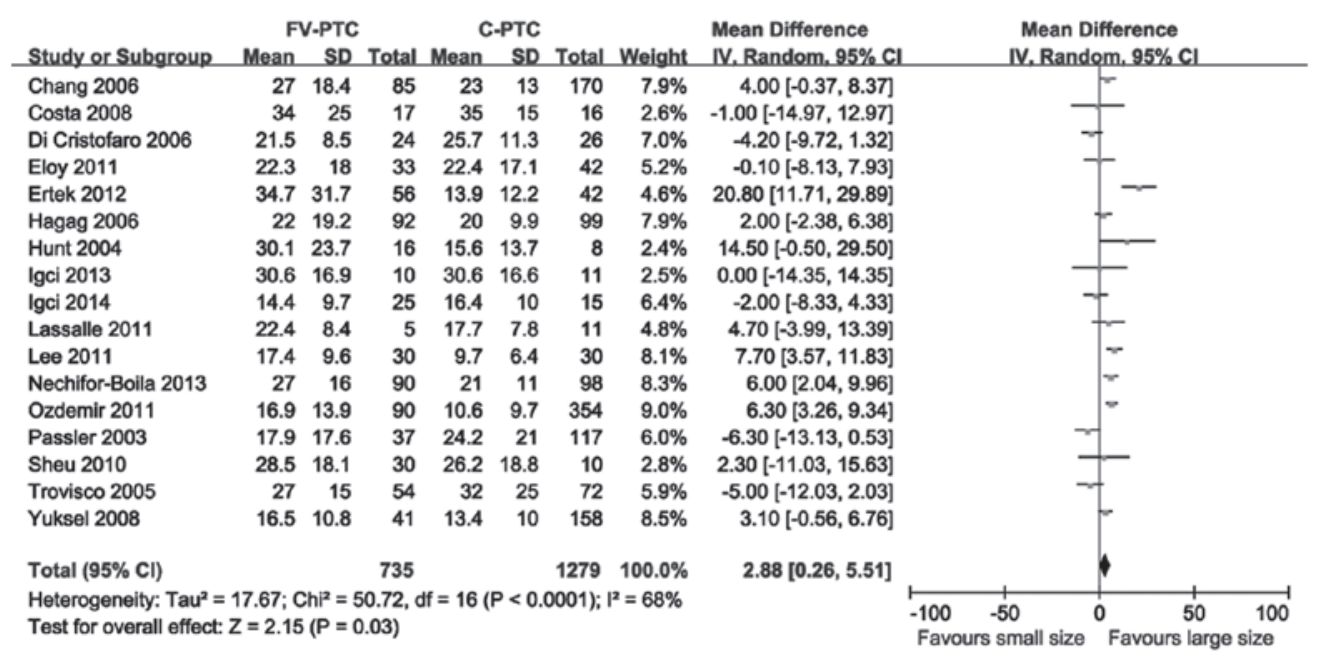

$\mathbf{E}$

FV-PTC

C-PTC

Odds Ratio

Odds Ratio

Study or Subgroup Events Total Events Total Weight M-H, Random, $95 \% \mathrm{Cl}$ M-H, Random, 95\% Cl

Abrosimov 2007

Eloy 2011

Gao 2012

Hunt 2004

Igci 2013

Igci 2014

Min 2013

Oler 2009

Ozdemir 2011

Slosar 2009

$\begin{array}{lllll}11 & 34 & 71 & 150 & 12.9 \%\end{array}$

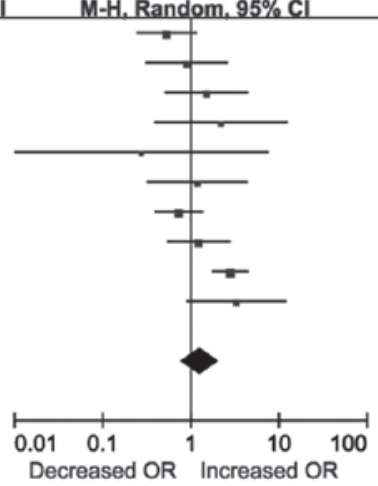

Total $(95 \% \mathrm{Cl})$

Total events

$\begin{array}{lllll}23 & 31 & 32 & 42 & 10.0 \%\end{array}$

$0.53[0.24,1.17]$

$0.90[0.31,2.63]$

$1.51[0.51,4.49]$

$2.20[0.38,12.57]$

$0.28[0.01,7.57]$

$1.19[0.32,4.42]$

$0.73[0.39,1.37]$

$1.23[0.53,2.81]$

$2.81[1.74,4.52]$

$3.27[0.88,12.06]$

Heterogeneity: $\mathrm{Tau}^{2}=0.31 ; \mathrm{Ch}^{2}=21.73, \mathrm{df}=9(P=0.010) ; 1^{2}=59 \%$

$1.25[0.77,2.03]$

Test for overall effect: $Z=0.92(P=0.36)$

$\mathbf{F}$

FV-PTC

C-PTC

\begin{tabular}{|c|c|c|c|c|c|c|c|c|c|}
\hline Study or Subgroup & Events & Io & Eve & Total & Neight & M-H, Random, $95 \% \mathrm{Cl}$ & \multicolumn{3}{|c|}{$\mathrm{M}-\mathrm{H}$, Random, $95 \% \mathrm{Cl}$} \\
\hline Burningham 2005 & 18 & 44 & 50 & 108 & $7.0 \%$ & $0.80[0.39,1.63]$ & & & \\
\hline Costa 2008 & 10 & 17 & 7 & 16 & $3.6 \%$ & $1.84[0.46,7.31]$ & & & \\
\hline Daglar-Aday 2013 & 11 & 36 & 17 & 72 & $5.8 \%$ & $1.42[0.58,3.48]$ & & & \\
\hline Eloy 2011 & 14 & 33 & 17 & 42 & $5.6 \%$ & $1.08[0.43,2.73]$ & & & \\
\hline Ertek 2012 & 24 & 56 & 13 & 42 & $6.1 \%$ & $1.67[0.72,3.88]$ & & & \\
\hline Gao 2012 & 5 & 25 & 38 & 84 & $4.9 \%$ & $0.30[0.10,0.88]$ & & & \\
\hline Igci 2013 & 4 & 10 & 5 & 11 & $2.6 \%$ & $0.80[0.14,4.53]$ & & & \\
\hline Igci 2014 & 20 & 25 & 7 & 15 & $3.5 \%$ & $4.57[1.12,18.73]$ & & & \\
\hline Lang 2006 & 20 & 67 & 97 & 308 & $7.9 \%$ & $0.93[0.52,1.65]$ & & & \\
\hline Lim 2013 & 10 & 85 & 885 & 2947 & $7.3 \%$ & $0.31[0.16,0.60]$ & & & \\
\hline Liu 2010 & 25 & 73 & 51 & 114 & $7.6 \%$ & $0.64[0.35,1.18]$ & & & \\
\hline Nechifor-Boila 2013 & 18 & 90 & 28 & 98 & $7.2 \%$ & $0.63[0.32,1.23]$ & & & \\
\hline Oler 2009 & 19 & 45 & 41 & 72 & $6.7 \%$ & $0.55[0.26,1.17]$ & & & \\
\hline Ozdemir 2011 & 30 & 90 & 102 & 354 & $8.4 \%$ & $1.24[0.75,2.03]$ & & & \\
\hline Passler 2003 & 12 & 37 & 17 & 117 & $6.0 \%$ & $2.82[1.20,6.67]$ & & & \\
\hline Trovisco 2005 & 13 & 39 & 22 & 35 & $5.5 \%$ & $0.30[0.11,0.77]$ & & & \\
\hline Yuksel 2008 & 4 & 41 & 14 & 158 & $4.4 \%$ & $1.11[0.35,3.58]$ & & & \\
\hline Total $(95 \% \mathrm{Cl})$ & & 813 & & 4593 & $100.0 \%$ & $0.88[0.64,1.22]$ & & & \\
\hline Total events & 257 & & 1411 & & & & & & \\
\hline \multicolumn{7}{|c|}{$\begin{array}{l}\text { Heterogeneity: Tau }{ }^{2}=0.26 ; \mathrm{Ch}^{2}=40.85, \text { df }=16(P=0.0006) ; I^{2}=61 \% \\
\text { Test for overall effect: } Z=0.77(P=0.44)\end{array}$} & $\begin{array}{ll}0.01 & 0.1 \\
\text { Decreased OR }\end{array}$ & $\begin{array}{ccc}1 & 1 & 1 \\
& 10 & 1 \\
\text { Increased OR }\end{array}$ & \\
\hline Study or Subaroup & $\begin{array}{l}\text { FV-PT } \\
\text { Events }\end{array}$ & $\begin{array}{l}\text { c } \\
\text { Total }\end{array}$ & $\begin{array}{l}\text { C-PTC } \\
\text { Events }\end{array}$ & Total & Weight & $\begin{array}{l}\text { Odds Ratio } \\
\text { M-H, Random. } 95 \% \mathrm{Cl}\end{array}$ & $\begin{array}{r}\text { Odds } \\
\text { M-H, Rand }\end{array}$ & $\begin{array}{l}\text { s Ratio } \\
\text { dom. } 95 \% \mathrm{Cl}\end{array}$ & \\
\hline Eloy 2011 & 12 & 33 & 14 & 42 & $14.5 \%$ & $1.14[0.44,2.97]$ & & & \\
\hline Ertek 2012 & 2 & 56 & 2 & 42 & $5.6 \%$ & $0.74[0.10,5.49]$ & & & \\
\hline Lang 2006 & 25 & 67 & 95 & 308 & $21.3 \%$ & $1.33[0.77,2.32]$ & & $=$ & \\
\hline Ozdemir 2011 & 14 & 90 & 98 & 354 & $20.2 \%$ & $0.48[0.26,0.89]$ & & & \\
\hline Rivera 2009 & 24 & 63 & 28 & 43 & $16.8 \%$ & $0.33[0.15,0.74]$ & & & \\
\hline Zidan 2003 & 38 & 100 & 44 & 143 & $21.6 \%$ & $1.38[0.81,2.36]$ & & - & \\
\hline Total $(95 \% \mathrm{Cl})$ & & 409 & & 932 & $100.0 \%$ & $0.82[0.48,1.39]$ & & & \\
\hline Total events & 115 & & 281 & & & & & & \\
\hline $\begin{array}{l}\text { Heterogeneity: Tau }{ }^{2}= \\
\text { Test for overall effect: }\end{array}$ & $\begin{array}{l}.26 ; \mathrm{Chi}^{2}= \\
=0.74(\mathrm{P}\end{array}$ & $\begin{array}{l}=14.60 \\
=0.46\end{array}$ & $d f=5(F$ & $(P=0.0$ & $01) ; 1^{2}=66$ & & $0.01 \quad 0.1$ & 10 & 100 \\
\hline
\end{tabular}

Figure 2. Continued. (D) Mean tumor size, (E) the tumor size being $\geq 10 \mathrm{~mm}$, (F) multifocality and (G) capsular invasion between follicular variant of papillary thyroid carcinoma (FV-PTC) and classical papillary thyroid carcinoma (C-PTC). ORs or MDs with corresponding 95\% CIs of individual studies for comparison of clinicopathological characteristics are shown. The forest plot shows the effect size and 95\% CIs for each study and overall. OR, odds ratio; MD, mean difference; $\mathrm{CI}$, confidence interval. 
H

FV-PTC C-PTC

Odds Ratio

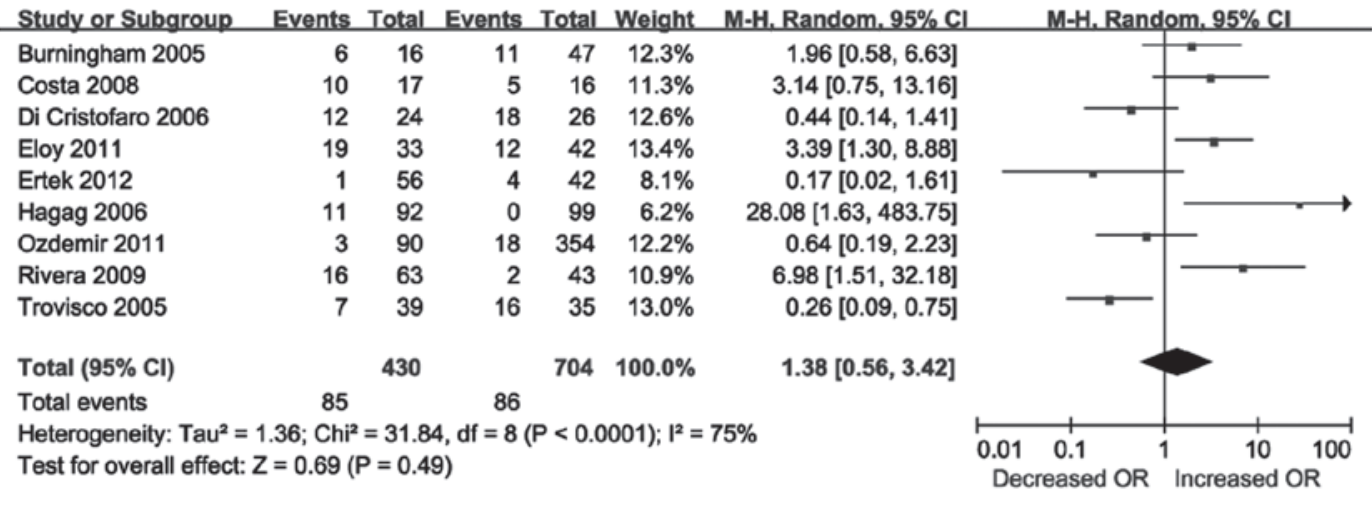

I

FV-PTC C-PTC

Odds Ratio

Odds Ratio

\begin{tabular}{|c|c|c|c|c|c|c|c|c|}
\hline Study or Subgroup & Events & Total & Events & Total & Weight & M-H. Random. $95 \% \mathrm{Cl}$ & M-H, Rano & dom. $95 \% \mathrm{Cl}$ \\
\hline Costa 2008 & 8 & 17 & 8 & 50 & $5.8 \%$ & $4.67[1.38,15.74]$ & & \\
\hline Eloy 2011 & 4 & 33 & 12 & 42 & $5.7 \%$ & $0.34[0.10,1.19]$ & & \\
\hline Ertek 2012 & 1 & 56 & 4 & 42 & $3.0 \%$ & $0.17[0.02,1.61]$ & & \\
\hline Gao 2012 & 4 & 25 & 19 & 84 & $5.9 \%$ & $0.65[0.20,2.13]$ & & - \\
\hline Hagag 2006 & 12 & 92 & 2 & 99 & $4.7 \%$ & $7.28[1.58,33.46]$ & & \\
\hline Hunt 2004 & 0 & 16 & 3 & 8 & $1.8 \%$ & $0.05[0.00,1.07]$ & & \\
\hline Igci 2014 & 1 & 25 & 1 & 15 & $2.1 \%$ & $0.58[0.03,10.08]$ & & \\
\hline Lang 2006 & 18 & 67 & 138 & 308 & $8.4 \%$ & $0.45[0.25,0.81]$ & $\rightarrow$ & \\
\hline Lim 2013 & 25 & 85 & 1587 & 2947 & $8.8 \%$ & $0.36[0.22,0.57]$ & $\rightarrow$ & \\
\hline Liu 2010 & 6 & 73 & 45 & 114 & $7.0 \%$ & $0.14[0.05,0.34]$ & & \\
\hline Min 2013 & 33 & 58 & 181 & 312 & $8.5 \%$ & $0.96[0.54,1.68]$ & & \\
\hline Nechifor-Boila 2013 & 7 & 90 & 28 & 98 & $7.2 \%$ & $0.21[0.09,0.51]$ & 5 & \\
\hline Oler 2009 & 7 & 45 & 33 & 69 & $7.0 \%$ & $0.20[0.08,0.51]$ & & \\
\hline Ozdemir 2011 & 7 & 90 & 58 & 354 & $7.4 \%$ & $0.43[0.19,0.98]$ & & \\
\hline Rivera 2009 & 0 & 63 & 2 & 43 & $1.9 \%$ & $0.13[0.01,2.79]$ & & \\
\hline Slosar 2009 & 7 & 60 & 12 & 37 & $6.5 \%$ & $0.28[0.10,0.78]$ & & \\
\hline Trovisco 2005 & 3 & 39 & 14 & 35 & $5.3 \%$ & $0.13[0.03,0.49]$ & & \\
\hline Wreesmann 2004 & 1 & 17 & 10 & 25 & $3.1 \%$ & $0.09[0.01,0.82]$ & & \\
\hline Total $(95 \% \mathrm{Cl})$ & & 951 & & 4682 & $100.0 \%$ & $0.40[0.25,0.64]$ & & \\
\hline Total events & 144 & & 2157 & & & & & \\
\hline $\begin{array}{l}\text { Heterogeneity: } \operatorname{Tau}^{2}=0 \\
\text { Test for overall effect: } 2\end{array}$ & $\begin{array}{l}.58 ; \mathrm{Chi}^{2} \\
=3.86(\mathrm{P}\end{array}$ & $\begin{array}{l}=56.75 \\
=0.00\end{array}$ & , df $=17$ & $(P<0$. & $.00001) ; 1^{2}$ & $=70 \%$ & $\begin{array}{l}0.01 \quad 0.1 \\
\text { Decreased OR }\end{array}$ & ${ }^{1} \underset{\text { Increased OR }}{10} 100$ \\
\hline Study or Subgroup & $\begin{array}{l}\text { FV-PT } \\
\text { Events }\end{array}$ & Iotal & $\begin{array}{l}\text { C-PTC } \\
\text { Events }\end{array}$ & Total & Weight & $\begin{array}{l}\text { Odds Ratio } \\
\text { M-H, Random, } 95 \% \text { Cl }\end{array}$ & $\begin{array}{r}\text { Odds } \\
\text { M-H, Rans }\end{array}$ & $\begin{array}{l}\text { s Ratio } \\
\text { dom, } 95 \% \mathrm{Cl}\end{array}$ \\
\hline Brzezianska 2007 & 4 & 8 & 3 & 14 & $2.3 \%$ & $3.67[0.56,24.13]$ & & \\
\hline Burningham 2005 & 4 & 46 & 13 & 114 & $4.0 \%$ & $0.74[0.23,2.40]$ & & - \\
\hline Costa 2008 & 12 & 17 & 12 & 16 & $2.9 \%$ & $0.80[0.17,3.73]$ & & \\
\hline Di Cristofaro 2006 & 7 & 24 & 19 & 26 & $3.8 \%$ & $0.15[0.04,0.52]$ & & \\
\hline Eloy 2011 & 8 & 33 & 19 & 42 & $4.6 \%$ & $0.39[0.14,1.05]$ & & \\
\hline Espadinha 2009 & 5 & 17 & 9 & 21 & $3.4 \%$ & $0.56[0.14,2.15]$ & & \\
\hline Gao 2012 & 5 & 20 & 45 & 84 & $4.2 \%$ & $0.29[0.10,0.87]$ & & \\
\hline Hagag 2006 & 29 & 92 & 30 & 99 & $6.1 \%$ & $1.06[0.57,1.96]$ & & \\
\hline Igci 2013 & 0 & 10 & 4 & 11 & $1.0 \%$ & $0.08[0.00,1.71]$ & & \\
\hline Ito 2008 & 60 & 100 & 1009 & 1313 & $6.8 \%$ & $0.45[0.30,0.69]$ & & \\
\hline Lang 2006 & 18 & 67 & 130 & 308 & $6.2 \%$ & $0.50[0.28,0.90]$ & & \\
\hline Lim 2013 & 13 & 85 & 1132 & 2947 & $6.2 \%$ & $0.29[0.16,0.53]$ & & \\
\hline Min 2013 & 18 & 58 & 248 & 312 & $6.1 \%$ & $0.12[0.06,0.22]$ & & \\
\hline Nechifor-Boila 2013 & 7 & 90 & 46 & 98 & $5.1 \%$ & $0.10[0.04,0.23]$ & & \\
\hline Oler 2009 & 10 & 47 & 41 & 73 & $5.2 \%$ & $0.21[0.09,0.49]$ & & \\
\hline Ozdemir 2011 & 6 & 90 & 29 & 354 & $4.9 \%$ & $0.80[0.32,1.99]$ & & \\
\hline Passler 2003 & 12 & 37 & 53 & 117 & $5.4 \%$ & $0.58[0.27,1.26]$ & & \\
\hline Rivera 2009 & 2 & 63 & 11 & 43 & $2.9 \%$ & $0.10[0.02,0.46]$ & & \\
\hline Slosar 2009 & 5 & 24 & 15 & 33 & $3.9 \%$ & $0.32[0.10,1.05]$ & & \\
\hline Trovisco 2005 & 6 & 39 & 23 & 35 & $4.2 \%$ & $0.09[0.03,0.29]$ & & \\
\hline Wreesmann 2004 & 4 & 17 & 12 & 25 & $3.4 \%$ & $0.33[0.08,1.31]$ & & \\
\hline Yuksel 2008 & 0 & 41 & 16 & 158 & $1.2 \%$ & $0.10[0.01,1.77]$ & & \\
\hline Zidan 2003 & 22 & 100 & 46 & 143 & $6.2 \%$ & $0.59[0.33,1.07]$ & & \\
\hline Total $(95 \% \mathrm{Cl})$ & & 1125 & & 6386 & $100.0 \%$ & $0.35[0.25,0.49]$ & 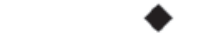 & \\
\hline Total events & 257 & & 2965 & & & & & \\
\hline \multicolumn{7}{|c|}{$\begin{array}{l}\text { Heterogeneity: } \text { Tau }^{2}=0.39 ; \mathrm{Chi}^{2}=65.94, \mathrm{df}=22(P<0.00001) ; I^{2}=67 \% \\
\text { Test for overall effect: } Z=6.12(P<0.00001)\end{array}$} & 0.01 & 10 \\
\hline
\end{tabular}

Figure 2. Continued. (H) Vascular invasion, (I) extrathyroidal extension and (J) lymph node metastasis between follicular variant of papillary thyroid carcinoma (FV-PTC) and classical papillary thyroid carcinoma (C-PTC). ORs or MDs with corresponding 95\% CIs of individual studies for comparison of clinicopathological characteristics are shown. The forest plot shows the effect size and 95\% CIs for each study and overall. OR, odds ratio; MD, mean difference; CI, confidence interval. 


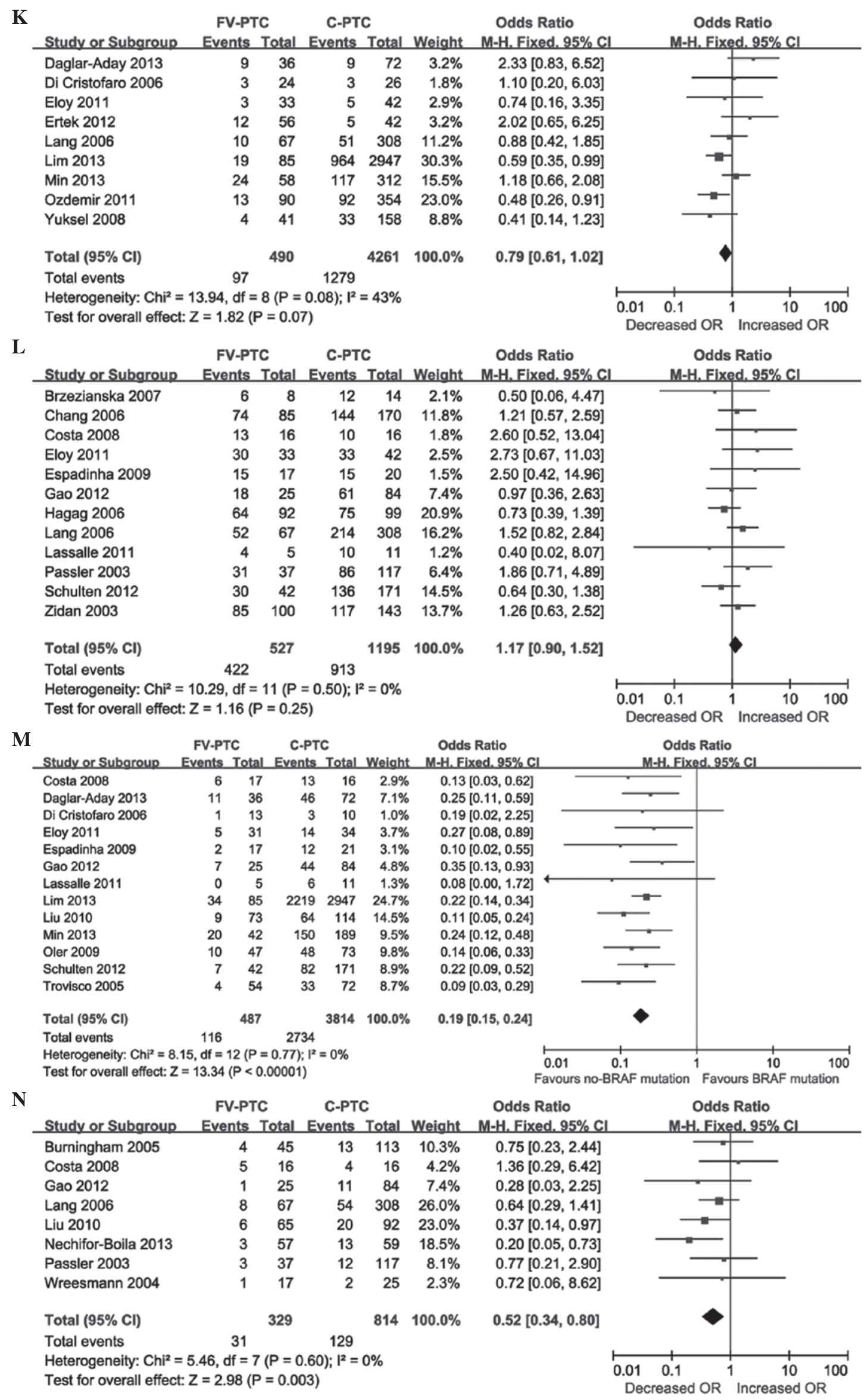

Figure 2. Continued. (K) Lymphocytic and/or Hashimoto's thyroiditis, (L) stages I + II, (M) BRAF mutation and (N) recurrence between follicular variant of papillary thyroid carcinoma (FV-PTC) and classical papillary thyroid carcinoma (C-PTC). ORs or MDs with corresponding 95\% CIs of individual studies for comparison of clinicopathological characteristics are shown. The forest plot shows the effect size and 95\% CIs for each study and overall. OR, odds ratio; MD, mean difference; CI, confidence interval. 
and C-PTC, which overall was 15.1 (144/951) and 46.1\% $(2,157 / 4,682)$, respectively, and the difference was statistically significant (OR, 0.40; 95\% CI, 0.25-0.64; P=0.0001; Fig. 2I). Significant statistical heterogeneity was detected among the studies $\left(\chi^{2}=56.75, \mathrm{P}<0.00001, \mathrm{I}^{2}=70 \%\right)$.

Lymph node metastasis. Twenty-three studies reported the prevalence of lymph node metastasis in patients with FV-PTC and C-PTC, which overall was $22.8(257 / 1,125)$ and $46.4 \%$ $(2,965 / 6,386)$, respectively, and this difference was statistically significant (OR, 0.35; 95\% CI, 0.25-0.49; P<0.00001; Fig. 2J). Significant statistical heterogeneity was detected among the studies $\left(\chi^{2}=65.94, \mathrm{P}<0.00001, \mathrm{I}^{2}=67 \%\right)$.

Lymphocytic and/or Hashimoto's thyroiditis. Nine studies presented the prevalence of lymphocytic and/or Hashimoto's thyroiditis in patients with FV-PTC and C-PTC. The overall prevalence was 19.8 (97/490) and 30.0\% (1,279/4,261), respectively, and the difference was not statistically significant (OR, 0.79; 95\% CI, 0.61-1.02; P=0.07; Fig. 2K). There was no significant statistical heterogeneity among the studies $\left(\chi^{2}=13.94, \mathrm{P}=0.08, \mathrm{I}^{2}=43 \%\right)$.

Clinical stage. Twelve studies included clinical stage in their analyses. The stage of the tumor was I or II in $422 / 527(80.1 \%)$ patients with FV-PTC and in 913/1,195 (76.4\%) patients with C-PTC; the difference was not statistically significant (OR, 1.17; 95\% CI, 0.0-1.52; P=0.25; Fig. 2L). There was no significant statistical heterogeneity among the studies $\left(\chi^{2}=10.29, \mathrm{P}=0.50, \mathrm{I}^{2}=0 \%\right)$.

$B R A F$ mutation. Thirteen studies presented the prevalence of $B R A F$ mutation in patients with FV-PTC and C-PTC, which overall was $23.8(116 / 487)$ and $71.7 \%(2,734 / 3,814)$, respectively, and the difference was statistically significant (OR, 0.19; 95\% CI, 0.15-0.24, P<0.00001; Fig. 2M). There was no significant statistical heterogeneity among the studies $\left(\chi^{2}=8.15, \mathrm{P}=0.77, \mathrm{I}^{2}=0 \%\right)$.

Recurrence. Eight studies evaluated the recurrence of tumor while following up patients with FV-PTC and C-PTC for varying periods. The overall percentage of recurrence was $9.4(31 / 329)$ and $15.8 \%$ (129/814), respectively, and the difference was statistically significant (OR, 0.52; 95\% CI, 0.34-0.80; $\mathrm{P}=0.003$; Fig. $2 \mathrm{~N}$ ). There was no significant statistical heterogeneity among the studies $\left(\chi^{2}=5.46, \mathrm{P}=0.60, \mathrm{I}^{2}=0 \%\right)$.

Publication bias. Funnel plots and Begg's test were performed to access the publication bias. All the Begg's funnel plots did not show evident asymmetry (Begg's funnel plots not shown), and the results of Egger's test were confirmed for the comparison of clinical parameters of FV-PTC and C-PTC (all P>0.05 for Egger's test; Table II). The results of Begg's funnel plot and Egger's test did not show any publication bias.

\section{Discussion}

Following a systematic review of the recent literature, it was observed that FV-PTC has been increasingly diagnosed in recent years, and an increasing amount of research is being performed concerning FV-PTC. The majority of studies comparing the clinicopathological behavior of FV-PTC and C-PTC have limitations, such as inclusion of relatively few cases, incomprehensive categories of clinical parameters and single-institution bias. Thus, their conclusions were mutually conflicting. Therefore, it is necessary to acquire a more comprehensive view of FV-PTC from population-based studies, and a meta-analysis can achieve this. To the best of our knowledge, this is the first study of a meta-analysis comparing the clinicopathological behavior of FV-PTV and C-PTC.

The findings reveal that the following clinicopathological parameters are significantly different between patients with FV-PTC and those with C-PTC: Patient age ( $\geq 45$ years), mean tumor size, extrathyroidal extension, lymph node metastasis, $B R A F$ mutation and recurrence. By contrast, no significant differences were identified in gender, mean age, tumor size ( $\geq 10 \mathrm{~mm}$ ), multifocality, capsular invasion, vascular invasion, lymphocytic and/or Hashimoto's thyroiditis, and clinical stage.

Patients with FV-PTC and C-PTC have similarly high prevalence of females, and this finding is consistent with nearly all the relevant studies. When analyzing age and tumor size, the meta-analysis was performed in two ways. The mean age of patients with FV-PTC was similar to that of patients with C-PTC, but the former were more likely to be $\geq 45$ years old. The number of patients with FV-PTC and C-PTC in the analysis of mean age was 656 and 972 (total 1,628) respectively, which is a larger population of patients in the analysis of age $\geq 45$ years being 219 and 371 (total 590). Logically, the mean age analysis may be more reliable. The findings were not consistent in the mean tumor size and the prevalence of tumor size $\geq 10 \mathrm{~mm}$. The mean tumor size of patients with FV-PTC was larger than that of the patients with C-PTC, which is in agreement with the results of studies by Chang et al (10), Ozdemir et al (17), Burningham et al (19), Jain et al (49) and Kim et al (50). Ozdemir et al (17) and Kim et al (50) also reported that compared to C-PTC, FV-PTC has more benign sonographic features, a lower incidence of a sonographically malignant grade and a lower diagnostic rate of PTC on fine-needle aspiration biopsy (FNAB). Thus, the lower rate of suspicious findings in FV-PTC lesions may have caused evaluation of larger FV-PTC lesions by FNAB, resulting in the detection of these lesions at a later stage. In addition, FV-PTC lesions may have become larger when patients with FV-PTC underwent surgery. However, the present study identified that the prevalence of tumor size $\geq 10 \mathrm{~mm}$ was similar between the two types. By contrast, Tielens et al (5) reported that tumor size of FV-PTC tends to be smaller than that of C-PTC. The present study showed similar prevalence of multifocality, capsular invasion, vascular invasion, lymphocytic and/or Hashimoto's thyroiditis, and clinical stage between patients with FV-PTC and C-PTC.

The prevalence of the above results has been controversial. Passler et al (12) suggested that there was a significantly higher prevalence of multifocality in patients with FV-PTC compared to patients with C-PTC, but the opposite was reported by Gao et al (31) and Trovisco et al (47). Certain studies reported a higher prevalence of capsular invasion and vascular invasion in the FV-PTC $(10,20,51)$, whereas others did not $(17,43,47)$. Although the majority of the C-PTCs do not have a tumor capsule, patients with C-PTC $(28.1 \%)$ and patients with 
FV-PTC (30.2\%) showed a similar high prevalence of capsular invasion of tumor in the present meta-analysis. As C-PTC is more inclined to show infiltrative growth, the tumor easily invades the capsule once containing the tumor capsule. When histopathologically comparing the presence of concomitant lymphocytic and/or Hashimoto's thyroiditis in patients with FV-PTC and C-PTC, Tielens et al (5) reported a higher rate in the former, whereas Yuksel et al (16) reported a higher rate in the latter.

Nearly all the relevant literature on clinical stages shows the same ratio of clinical stage I + II in patients with FV-PTC and C-PTC, thereby suggesting that clinical stages of patients with FV-PTC and C-PTC are similar. The present meta-analysis reveals that the incidence of associated extrathyroidal extension, lymph node metastases, $B R A F$ mutation and recurrence is significantly lower in patients with FV-PTC compared to patients with C-PTC. As described in the majority of the studies, the frequencies of extrathyroidal extension and lymph node metastases are lower in patients with FV-PTC compared to patients with C-PTC; however, a few studies reported opposing findings $(20,24)$. Consistently, all the relevant studies on the $B R A F$ gene shows the lower ratio of $B R A F$ mutation in patients with FV-PTC compared to patients with C-PTC. The majority of meta-analysis studies showed that the BRAF mutation was associated with the majority of vital clinicopathological characteristics in PTC, and the BRAF mutation may be used as an important prognostic marker of patients with PTC (52-56). However, when analyzing FV-PTC and C-PTC respectively, Gao et al (31) and Oler et al (42) identified that the BRAF mutation was associated with the clinicopathological characteristics in patients with C-PTC, but not in patients with FV-PTC.

After the similar follow-up periods, the rate of recurrence in patients with FV-PTC was significantly lower than that in the patients with C-PTC, which may be associated with the above finding that patients with FV-PTC are at a lower risk of extrathyroidal extension, lymph node metastases or $B R A F$ mutation. Aggressive clinicopathological behavior of patients with PTC is associated with old age, and include the following: Presence of extrathyroidal extension, lymph node metastases, advanced clinical stages and $B R A F$ mutation. Therefore, poor prognosis in patients with PTC is associated with certain aggressive clinicopathological characteristics. Therefore, patients with FV-PTC have an improved prognosis compared to patients with C-PTC. However, it may be affected by treatment factors such as type of surgery, I-131 ablation and use of external radiotherapy.

Patients with FV-PTC have a lower prevalence of extrathyroidal extension, lymph node metastases, $B R A F$ mutation and recurrence compared to patients with C-PTC. The mean tumor size is larger and the incidence of patients aged $\geq 45$ years are higher in the former. Thus, as reported in a previous study (8) and in the present meta-analysis, patients with FV-PTC exhibit a more favorable clinicopathological behavior and improved prognosis compared to patients with C-PTC. Thus, the lower incidence of extrathyroidal extension, lymph node metastases, $B R A F$ mutation and recurrence would appear to be distinct clinicopathological behavior of FV-PTC. Patients with FV-PTC and C-PTC are clearly two different groups. As was also reported by Yu et al (57) and Chang et al (10), the clinicopathological behavior of FV-PTC is unique and represents an intermediate entity between C-PTC and FTC. Different approaches may be used for their clinical management. More invasive treatment strategies, such as total thyroidectomy or central lymph node dissection, may be considered in patients with C-PTC presenting extrathyroidal extension, lymph node metastasis or $B R A F$ mutation to decrease recurrence.

The present study has several limitations. One primary limitation is that reporting of FV-PTC is not a standard practice in certain hospitals, leading to a reporting bias. There may be an interpretational difference among pathologists, as FV-PTC may be confused with FTC. Additionally, stratified analyses of summary data from the reported studies could not be performed, and the present study was unable to identify the diverse sources of heterogeneity of the effect size. In addition, multiple outcome variables require cautious interpretation as the outcomes may be interrelated. For instance, the patients with extrathyroidal extension tend to have a more advanced clinical stage and a higher risk of recurrence than those with no extension. However, this limitation is unlikely to have a significant impact on the present study as the sample size in the meta-analysis is sufficiently large.

The meta-analysis suggested that, patients with FV-PTC present more favorable clinicopathological behaviors and improved prognosis than patients with C-PTC. Patients with FV-PTC and C-PTC may be managed differently, and the two types of PTC should be clearly distinguished in future retrospective or prospective studies. For instance, it may not be necessary for patients with FV-PTC to undertake the invasive strategies that are appropriate in patients with C-PTC, if the FV-PTC is diagnosed prior to or during surgery. However, more valuable studies on a large cohort of cases are required to evaluate the clinicopathological behavior in patients with FV-PTC and patients with C-PTC.

\section{Acknowledgements}

The present study was supported by a grant from the Department of Sichuan Province, Science and Technology Support Program (no. 2013SZ0041).

\section{References}

1. Howlader N, Noone AM, Krapcho M, Garshell J, Miller D, Altekruse SF, Kosary CL, Yu M, Ruhl J, Tatalovich Z, Mariotto A, Lewis DR, Chen HS, Feuer EJ and Cronin KA (eds): SEER Cancer Statistics Review, 1975-2011, National Cancer Institute, Bethesda, MD. http://seer.cancer.gov/csr/1975_2011/. Access date September 17, 2014.

2. Sipos JA and Mazzaferri EL: Thyroid cancer epidemiology and prognostic variables. Clin Oncol (R Coll Radiol) 22: 395-404, 2010.

3. Carcangiu ML, Zampi G, Pupi A, Castagnoli A and Rosai J: Papillary carcinoma of the thyroid. A clinicopathologic study of 241 cases treated at the University of Florence, Italy. Cancer 55: 805-828, 1985.

4. Albores-Saavedra $\mathrm{J}$ and $\mathrm{Wu} \mathrm{J}$ : The many faces and mimics of papillary thyroid carcinoma. Endocr Pathol 17: 1-18, 2006.

5. Tielens ET, Sherman SI, Hruban RH and Ladenson PW: Follicular variant of papillary thyroid carcinoma: a clinicopathologic study. Cancer 73: 424-431, 1994.

6. Sebastian SO, Gonzalez JM, Paricio PP, Perez JS, Flores DP Madrona AP, Romero PR and Tebar FJ: Papillary thyroid carcinoma: prognostic index for survival including the histological variety. Arch Surg 135: 272-277, 2000.

7. Zidan J, Karen D, Stein M, Rosenblatt E, Basher W and Kuten A: Pure versus follicular variant of papillary thyroid carcinoma: clinical features,prognostic factors, treatment and survival. Cancer 97: 1181-1185, 2003. 
8. Lang BH, Lo CY, Chan WF, Lam AK and Wan KY: Classical and follicular variant of papillary thyroid carcinoma: a comparative study on clinicopathologic features and long-term outcome. World J Surg 30: 752-758, 2006.

9. Lam AK, Lo CY and Lam KS: Papillary carcinoma of thyroid: A 30-yr clinicopathological review of the histological variants. Endocr Pathol 16: 323-330, 2005.

10. Chang HY, Lin JD, Chou SC, Chao TC and Hsueh C: Clinical presentations and outcomes of surgical treatment of follicular variant of the papillary thyroid carcinomas. Jpn J Clin Oncol 36: 688-693, 2006

11. Lin HW and Bhattacharyya N: Clinical behavior of follicular variant of papillary thyroid carcinoma: presentation and survival. Laryngoscop 120: 712-716, 2010.

12. Passler C, Prager G, Scheuba C, Niederle BE, Kaserer K, Zettinig G and Niederle B: Follicular variant of papillary thyroid carcinoma: a long-term follow-up. Arch Surg 138: 1362-1366, 2003.

13. Crile GJ and Hazard JB: Relationship of the age of the patient to the natural history and prognosis of carcinoma of the thyroid Ann Surg 138: 33-38, 1953.

14. Ertek S, Yilmaz NC, Cicero AF, Vurupalmaz O, Demiroz AS and Erdogan G: Increasing diagnosis of thyroid papillary carcinoma follicular variant in south-east Anatolian region: comparison of characteristics of classical papillary and follicular variant thyroid cancers. Endocr Pathol 23: 157-160, 2012.

15. LiVolsi VA and Asa SL: The demise of follicular carcinoma of the thyroid gland. Thyroid 4: 233-236, 1994.

16. Yuksel O, Kurukahvecioglu O, Ege B, Ekinci O, Aydin A, Poyraz A, Tezel E and Taneri F: The relation between pure papillary and follicular variant in papillary thyroid carcinoma. Endocr Regul 42: 29-33, 2008.

17. Ozdemir D, Ersoy R, Cuhaci N, Arpaci D, Ersoy EP, Korukluoglu B, Guler G and Cakir B: Classical and follicular variant papillary thyroid carcinoma: comparison of clinical, ultrasonographical, cytological and histopathological features in 444 patients. Endocr Pathol 22: 58-65, 2011.

18. Cheng S, Serra S, Mercado M, Ezzat S and Asa SL: A high-throughput proteomic approach provides distinct signatures for thyroid cancer behavior. Clin Cancer Res 17: 2385-2394, 2011

19. Burningham AR, Krishnan J, Davidson BJ, Ringel MD and Burman KD: Papillary and follicular variant of papillary carcinoma of the thyroid: Initial presentation and response to therapy. Otolaryngol Head Neck Surg 132: 840-844, 2005.

20. Hagag P, Hod N, Kummer E, Cohenpour M, Horne T and Weiss M: Follicular variant of papillary thyroid carcinoma: clinical-pathological characterization and long-term follow-up. Cancer J 12: 275-282, 2006.

21. Stroup DF, Berlin JA, Morton SC, Olkin I, Williamson GD, Rennie D, Moher D, Becker BJ, Sipe TA and Thacker SB: Meta-analysis of observational studies in epidemiology: a proposal for reporting. Meta-analysis of observational studies in epidemiology (MOOSE) group. JAMA 283: 2008, 2012.

22. Abrosimov A, Saenko V, Meirmanov S, Nakashima M Rogounovitch T, Shkurko O, Lushnikov E, Mitsutake N, Namba H and Yamashita S: The cytoplasmic expression of MUC1 in papillary thyroid carcinoma of different histological variants and its correlation with cyclin D1 overexpression. Endocr Pathol 18: 68-75, 2007.

23. Brzezianska E, Pastuszak-Lewandoska D, Wojciechowska K, Migdalska-Sek M, Cyniak-Magierska A, Nawrot E and Lewinski A: Investigation of V600E BRAF mutation in papillary thyroid carcinoma in the Polish population. Neuro Endocrinol Lett 28: 351-359, 2007.

24. Costa AM, Herrero A, Fresno MF, Heymann J, Alvarez JA, Cameselle-Teijeiro J and Garcia-Rostan G: BRAF mutation associated with other genetic events identifies a subset of aggressive papillary thyroid carcinoma. Clin Endocrinol (Oxf) 68: 618-634, 2008.

25. Daglar-Aday A, Toptas B, Ozturk T, Seyhan F, Saygili N, Eronat AP, Akadam-Teker B, Yilmaz-Aydogan H, Aksoy F and Ozturk O: Investigation of BRAF V600E mutation in papillary thyroid carcinoma and tumor-surrounding nontumoral tissues. DNA Cell Biol 32: 13-18, 2013.

26. Darr EA, Patel AD, Yu G, Komorowski Z, McCormick S, Tiwari R, Schantz SP and Geliebter J: Reduced Cx43 gap junction plaque expression differentiates thyroid carcinomas from benign disease. Arch Otolaryngol Head Neck Surg 137: 1161-1165, 2011.

27. Dettmer M, Perren A, Moch H, Komminoth P, Nikiforov YE and Nikiforova MN: Comprehensive MicroRNA expression profiling identifies novel markers in follicular variant of papillary thyroid carcinoma. Thyroid 23: 1383-1389, 2013.
28. Di Cristofaro J, Marcy M, Vasko V, Sebag F, Fakhry N, Wynford-Thomas D and De Micco C: Molecular genetic study comparing follicular variant versus classic papillary thyroid carcinomas: association of N-ras mutation in codon 61 with follicular variant. Hum Pathol 37: 824-830, 2006.

29. Eloy C, Santos J, Soares P and Sobrinho-Simoes M: The preeminence of growth pattern and invasiveness and the limited influence of BRAF and RAS mutations in the occurrence of papillary thyroid carcinoma lymph node metastases. Virchows Arch 459: 265-276, 2011.

30. Espadinha C, Santos JR, Sobrinho LG and Bugalho MJ: Expression of iodine metabolism genes in human thyroid tissues: evidence for age and BRAFV600E mutation dependency. Clin Endocrinol (Oxf) 70: 629-635, 2009.

31. Gao WL, Wie LL, Chao YG, Wie L and Song TL: Prognostic prediction of BRAF (V600E) and its relationship with sodium iodide symporter in classic variant of papillary thyroid carcinomas. Clin Lab 58: 919-926, 2012

32. Hunt JL, Fowler M, Lomago D, Niehouse L, Sasatomi E, Swalsky P and Finkelstein S: Tumor suppressor gene allelic loss profiles of the variants of papillary thyroid carcinoma. Diagn Mol Pathol 13: 41-46, 2004.

33. Igci YZ, Erkilic S, Igci M and Arslan A: MCM3 Protein expression in follicular and classical variants of papillary thyroid carcinoma. Pathol Oncol Res 20: 87-91, 2013.

34. Igci YZ, Erkilic S and Arslan A: Septin 7 immunoexpression in papillary thyroid carcinoma: a preliminary study. Pathol Res Pract 210: 426-431, 2014.

35. Ito $Y$, Hirokawa M, Uruno T, Kihara M, Higashiyama $T$, Takamura Y, Miya A, Kobayashi K, Matsuzuka F and Miyauchi A: Prevalence and biological behaviour of variants of papillary thyroid carcinoma: experience at a single institute. Pathology 40: 617-622, 2008

36. Lassalle S, Hofman V, Ilie M, Bonnetaud C, Puissegur MP, Brest P, Loubatier C, Guevara N, Bordone O, Cardinaud B, Lebrigand K, Rios G, Santini J, Franc B, Mari B, Al Ghuzlan A, Vielh P, Barbry P, and Hofman P: Can the microRNA signature distinguish between thyroid tumors of uncertain malignant potential and other well-differentiated tumors of the thyroid gland? Endocr Relat Cancer 18: 579-594, 2011

37. Lee S, Han BK, Ko EY, Oh YL, Choe JH and Shin JH: The ultrasonography features of hyalinizing trabecular tumor of the thyroid are more consistent with its benign behavior than cytology or frozen section readings. Thyroid 21: 253-259, 2011.

38. Lim JY, Hong SW, Lee YS, Kim BW, Park CS, Chang HS and Cho JY: Clinicopathologic implications of the BRAF (V600E) mutation in papillary thyroid cancer: a subgroup analysis of 3130 cases in a single center. Thyroid 23: 1423-1430, 2013.

39. Liu Z, Liu D, Bojdani E, El-Naggar AK, Vasko V and Xing M: IQGAP1 plays an important role in the invasiveness of thyroid cancer. Clin Cancer Res 16: 6009-6018, 2010.

40. Min HS, Lee C and Jung KC: Correlation of immunohistochemical markers and BRAF mutation status with histological variants of papillary thyroid carcinoma in the Korean population. J Korean Med Sci 28: 534-541, 2013.

41. Nechifor-Boila A, Borda A, Sassolas G, Hafdi-Nejjari Z, Borson-Chazot F, Lifante J, C, Sturm N, Laverriere MH, Berger $\mathrm{N}$ and Decaussin-Petrucci M: Immunohistochemical markers in the diagnosis of papillary thyroid carcinomas: The promising role of combined immunostaining using HBME-1 and CD56. Pathol Res Pract 209: 585-592, 2013.

42. Oler G and Cerutti JM: High prevalence of BRAF mutation in a Brazilian cohort of patients with sporadic papillary thyroid carcinomas: correlation with more aggressive phenotype and decreased expression of iodide-metabolizing genes. Cancer 115: 972-980, 2009

43. Rivera M, Tuttle RM, Patel S, Shaha A, Shah JP and Ghossein RA: Encapsulated papillary thyroid carcinoma: a clinico-pathologic study of 106 cases with emphasis on its morphologic subtypes (histologic growth pattern). Thyroid 19: 119-127, 2009.

44. Schulten HJ, Salama S, Al-Mansouri Z, Alotibi R, Al-Ghamdi K, Al-Hamour OA, Sayadi H, Al-Aradati H, Al-Johari A, Huwait E Gari M, Al-Qahtani MH and Al-Maghrabi J: BRAF mutations in thyroid tumors from an ethnically diverse group. Hered Cancer Clin Pract 10: 10, 2012.

45. Sheu SY, Grabellus F, Schwertheim S, Worm K, Broecker-Preuss $M$ and Schmid KW: Differential miRNA expression profiles in variants of papillary thyroid carcinoma and encapsulated follicular thyroid tumours. Br J Cancer 102: 376-382, 2010 
46. Slosar M, Vohra P, Prasad M, Fischer A, Quinlan R and Khan A Insulin-like growth factor mRNA binding protein 3 (IMP3) is differentially expressed in benign and malignant follicular patterned thyroid tumors. Endocr Pathol 20: 149-157, 2009.

47. Trovisco V, Soares P, Preto A, de Castro IV, Lima J, Castro P, Maximo V, Botelho T, Moreira S, Meireles AM, Magalhaes J, Abrosimov A, Cameselle-Teijeiro J and Sobrinho-Simoes M: Type and prevalence of BRAF mutations are closely associated with papillary thyroid carcinoma histotype and patients' age but not with tumour aggressiveness. Virchows Arch 446: 589-595, 2005.

48. Wreesmann VB, Ghossein RA, Hezel M, Banerjee D, Shaha AR Tuttle RM, Shah JP, Rao PH and Singh B: Follicular variant of papillary thyroid carcinoma: genome-wide appraisal of a controversial entity. Genes Chromosomes Cancer 40: 355-364, 2004.

49. Jain M, Khan A, Patwardhan N, Reale F and Safran M: Follicular variant of papillary thyroid carcinoma: a comparative study of histopathologic features and cytology results in 141 patients. Endocr Pract 7: 79-84, 2001.

50. Kim DS, Kim JH, Na DG, Park SH, Kim E, Chang KH, Sohn CH and Choi YH: Sonographic features of follicular variant papillary thyroid carcinomas in comparison with conventional papillary thyroid carcinomas. J Ultrasound Med 28: 1685-1692, 2009.

51. Salajegheh A, Petcu EB, Smith RA and Lam AK: Follicular variant of papillary thyroid carcinoma: a diagnostic challenge for clinicians and pathologists. Postgrad Med J 84: 78-82, 2008.
52. Lee JH, Lee ES and Kim YS: Clinicopathologic significance of BRAF V600E mutation in papillary carcinomas of the thyroid: a meta-analysis. Cancer 110: 38-46, 2007.

53. Kim TH, Park YJ, Lim JA, Ahn HY, Lee EK, Lee YJ, Kim KW, Hahn SK, Youn YK, Kim KH, Cho BY and Park do J: The association of the BRAF (V600E) mutation with prognostic factors and poor clinical outcome in papillary thyroid cancer: a meta-analysis. Cancer 118: 1764-1773, 2012.

54. Tufano RP, Teixeira GV, Bishop J, Carson KA and Xing M: BRAF mutation in papillary thyroid cancer and its value in tailoring initial treatment: a systematic review and meta-analysis. Medicine (Baltimore) 91: 274-286, 2012.

55. Li C, Lee KC, Schneider EB and Zeiger MA: BRAF V600E mutation and its association with clinicopathological features of papillary thyroid cancer: a meta-analysis. J Clin Endocrinol Metab 97: 4559-4570, 2012.

56. Liu X, Yan K, Lin X, Zhao L, An W, Wang C and Liu X: The association between BRAF V600E mutation and pathological features in PTC. Eur Arch Otorhinolaryngol 271: Jan 4, 2014 (Epub ahead of print).

57. Yu XM, Schneider DF, Leverson G, Chen H and Sippel RS: Follicular variant of papillary thyroid carcinoma is a unique clinical entity: a population-based study of 10,740 cases. Thyroid 23: 1263-1268, 2013. 